\title{
The role of colchicine treatment on reproductive outcome in women with Familial Mediterranean Fever
}

\author{
(D)Arzu Yurci ${ }^{1}$, @Nurettin Türktekin ${ }^{2}$ \\ ${ }^{1}$ Memorial Kayseri Hospital, Department of Gynecology Obstetrics and Reproductive Medicine, IVF Center, Kayseri, Turkey \\ ${ }^{2}$ İstanbul IVF Center, Department of Gynecology and Obstetrics, IVF Center, İstanbul, Turkey
}

Cite this article as: Yurci A, Türktekin N. The role of colchicine treatment on reproductive outcome in women with Familial Mediterranean Fever. J Health Sci Med 2021; 4(3): 283-288.

\begin{abstract}
Aim: This study was planned to compare the reproductive outcome of women diagnosed with Familial Mediterranean Fever (FMF) who were given colchicine treatment and those who were not.

Material and Method: Forty infertile women with FMF were divided into two groups whether they received colchicine treatment or not. While 29 FMF patients received regular colchicine treatment 11 patients did not receive colchicine treatment. The patients were diagnosed with FMF before applying to our IVF clinic. All patients were subjected to full infertility work-up, including hormonal profile, semen analysis and hysterosalpingography (HSG). All women with FMF were treated according to a standard antagonist protocol. The primary outcome measures were the percantage of clinical pregnancy (CPR), miscarraige rates, ongoing pregnancy, live birth and term birth rates.

Results: We detected three types of problems in HSG; (i) peritubal adhesion (ii) periovarian adhesion and (iii) hydrosalpinx. Eleven patients underwent laparoscopy due to abnormal findings in HSG. Adhesion was detected in 8 cases in the colchicine group, while adhesion was detected in 3 cases in the patients who were not given colchicine. Hydrosalpinx was detected in 8 cases in the colchicine group, while it was detected in only one case in those who did not take colchicine. Ascites in the Douglas pouch was detected ultrasonographically or by laparoscopy in both groups. The CPR was found to be significantly higher in patients who did not receive colchicine compared to patients who received colchicine. There was no significant difference between two groups in terms of miscarraige rates. While preterm birth rates were found to be significantly lower in the colchicine group term birth rates were significantly higher in colchicine group compared to control group.

Conclusion: Except for the positive effect on the number of term pregnancies we could not detect any negative effect of the colchicine treatment on the reproductive outcome.
\end{abstract}

Keywords: FMF, colchicine, birth rates, miscarriage rates

\section{INTRODUCTION}

Familial Mediterranean fever (FMF) is a hereditary disease characterized by recurrent episodes of fever, peritonitis, pleuritis or arthritis. The most important complication of FMF is amyloid deposition primarily involving the kidneys (1). Although we do not have very clear scientific data, there is a common belief that FMF causes subfertility in both men and women. It is accepted that FMF might cause subfertility by disrupting sperm count and movement in men. On the other hand, in women diagnosed with FMF, peritoneal/peritubal adhesions, ovulatory dysfunction and exudate accumulating in the douglos space, which are believed to develop due to recurrent FMF attacks, are considered to be the cause of subfertility. In some infertile FMF cases, the presence of hydroslapinx has been reported as a cause of subfertility. Another view is that amyloid accumulation, which is one of the long-term complications of FMF, disrupts sperm functions in the testicles and oocyte development in the ovaries and causes subfertility $(2,3)$. If we need to elaborate, it has been experimentally shown that oocytes and sperm exposed to amyloid deposition fail to achieve spontaneous fertilization. However, it is a known fact that amyloid accumulation is a problem rarely encountered in both gonad types $(3,4)$ Therefore, subfertility due to amyloid accumulation in FMF cases can be considered as a negligible etiological cause. 
Another important reason blamed as the cause of subfertility in FMF patients is the use of colchicine. Colchicine is an alkaloid whose main mechanism of action is to block microtubule formation. Because of this effect, colchicine has a negative effect on cells that undergo both mitosis and meiosis (5). Colchicine prevents spindle formation by blocking microtubule formation and may cause both spermatogenesis and folliculogenesis to deviate from normal. However, the clinical and laboratory data we have do not support this idea (6). Although colchicine is claimed to cause different sperm analyzes from oligospermia to azoospermia, most of the available data do not support these negative effects of the drug. Many independent authors concluded that colchicine have no significant direct adverse effect on sperm production and function (7).

Similar to its effects on spermatagonesis, the effects of colchicine on oocyte development and function in women are controversial $(3,8)$ The main purpose of colchicine use in the treatment of FMF, Behcet's disease and gouty arthritis is its anti-inflammatory effect, not its blockage on microtubules (9). For all reasons, an infertile woman using colchicine diagnosed with FMF should have less subfertility complaints due to the antiinflammatory effect of the drug. As a result of the decrease in peritoneal, peritubal and periovarian adhesions due to the anti-inflammatory effect of the drug, FMF patients need to conceive more easily both spontaneously and in IVF-ET cycles. When the literature is reviewed, it has been reported that the IVF-ET results of FMF patients using colchicine are similar to the general population. So far, there are no studies showing the effect of colchicine use or not on IVF-ET outcomes in FMF patients. This study was planned to compare the IVF-ET results of women diagnosed with FMF who were given colchicine treatment and those who were not.

\section{MATERIAL AND METHOD}

The study was carried out with the permission of the Research Ethics Committee of Erciyes University (Date: 09/09/2020, Decision No: 2020-426). This study was conducted in accordance with the Declaration of Helsinki and written informed consent was obtained from all participants at the time of enrollment. Forty FMF patients who applied to Erciyes University with infertility complaints were included in the study.

The patients were divided into two groups according to whether they received colchicine treatment or not. While 29 FMF patients received regular colchicine treatment from the time of diagnosis, 11 patients did not receive regular colchicine treatment. Some of the patients in the second group received short-term colchicine treatment during FMF attacks, but they did not use colchicine for the last two years. The patients were diagnosed with FMF before applying to our IVF clinic. They were diagnosed as having FMF when they had: (a) at least four episodes of abdominal or chest pain or both, lasting from 24-72 h; (b) lack of symptoms between FMF attacks; (c) no other condition that would explain the symptoms; $(\mathrm{d})$ a response to colchicine treatment; and (e) a positive MEFV gene for FMF mutation. Before their admission to us, there was a history of laparoscopy in seven cases whose FMF attacks were accepted as acute abdomen, and laparotomy in one case. Six cases in treatmetn group and two cases in control group had history of appendectomy. All patients were subjected to full infertility work-up, including hormonal profile, hysterosalpingography (HSG), measurement of serum progesterone and semen analysis. Antral follicle counts were also noted in all participants. Patients with male factor infertility and couples with FMF in their male partners were not included in the study. In addition, patients using colchicine for Behçet's disease or gouty arthritis were not included in the study. Patients in the colchicine group continued to receive their treatments before and after embryo transfer. Participants in the treatment group had been receiving colchicine twice a day for approximately $11.10 \pm 3.51$ years (Colchium dispert $0.5 \mathrm{mg}$, Recordati, Turkey). Some patients received antiinflammatory or corticosteroid treatments in addition to colchicine treatment during the attack periods.

All women with FMF were treated according to a standard antagonist protocol with individually dosed recombinant FSH starting on day 2-3 of the menstrual cycle. Gonadotrophin-releasing hormone antagonist was started on the 5th or 6th day of stimulation. When at least three follicles reached $16-17 \mathrm{~mm}$ in diameter, maturation of follicles was induced with recombinant hCG. Oocyte collection was performed 36 hours after hCG application. Ovarian follicles were aspirated using a single-lumen, 17-gauge needle guided by trans-vaginal ultrasonography. The primary outcome measures were the percantage of clinical pregnancy, miscarraige rates, ongoing pregnancy, live birth and term birth rates. The birth weights of all newborn were also noted.

\section{Statistical Analysis}

Statistical analyses were performed with the SPSS v21 (SPSS Inc., Chicago, IL, USA). The normality distribution of data was tested with the use of the KolmogorovSmirnov test, and all variables were skewed normally. The continuous variables were analyzed by means of analysis of variance test with posthoc Tukey procedure and Mann-Whitney $U$ test. The categorical data were analyzed by means of the Pearson chi-square test. A P value of $<.05$ was considered to be significant. The results are expressed as mean $\pm \mathrm{SD}$ or percentage. 


\section{RESULTS}

The mean age of the patients included in the study from both groups was found to be similar $(32.07 \pm 4.85$ vs $31.12 \pm 1.01)$. Infertility duration, BMI and the number of aIVF-ET attempt of both groups were recorded as similar. The clinical, laboratory and hormonal profiles of the patients regarding IVF-ET are shown in detail in Table 1. There was no significant difference between the basal hormone and pre-OPU hormonal evaluations of the patients. While 22 of 29 cases in the colchicine group were primary infertility, 7 cases were secondary infertility. In patients who did not take colchicine, 7 patients were recorded as primary infertility, while 4 patients were recorded as secondary infertility. While 9 patients in the colchicine group had a history of abortion, 2 patients in the control group had a history of abortion. While 6 of the patients who received colchicine had a history of appendectomy, 2 patients in the control group had a history of appendectomy. In summary, before applying for IVF-ET, a history of surgical intervention was detected in 8 patients from both groups. The number of patients who underwent laparoscopy due to HSG pathology was 8 in the colchicine group and 3 in the control group. Diagnostic or therapeutic laparoscopy was performed in 11 of 40 patients in both groups.

We detected three types of different problems in HSG; (i) peritubal adhesion (ii) periovarian adhesion and (iii) hydrosalpinx. Eleven patients underwent laparoscopy due to abnormal findings in HSG. While 8 of the cases were in the group receiving colchicine 3 of them were in the patient group not receiving colchicine. Adhesion was detected in 8 cases (27.5\%) in the colchicine group, while adhesion was detected in 3 cases $(27.2 \%)$ in the patients who were not given colchicine. Hydrosalpinx was detected in 8 cases in the colchicine group, while it was detected in only one case in those who did not take colchicine. While salpingectomy was performed in hydrosalpinx cases, adhesiolysis was performed in the presence of adhesion. In one case, hydrosalpinx due to unilateral fimbrial phimosis was detected and corrected. Detailed anamnesis of 3 of the cases with hydrosalpinx were found to have previously undergone appendectomy (Table 2).

\section{Table 1. Demographic and clinical charactersitics of both groups of participants}

\begin{tabular}{|c|c|c|}
\hline & $\begin{array}{l}\text { FMF patients treated with colchicine } \\
\qquad(\mathbf{n}=29)\end{array}$ & $\begin{array}{l}\text { FMF patients not given colchicine treatment } \\
\qquad(\mathrm{n}=11)\end{array}$ \\
\hline Age (year) & $32.07 \pm 4.85$ & $31.12 \pm 1.01$ \\
\hline BMI $\left(\mathrm{kg} / \mathrm{m}^{2}\right)$ & $23.97 \pm 3.69$ & $24.12 \pm 2.14$ \\
\hline Infertility duration (month) & $65.72 \pm 43.26$ & $63.81 \pm 21.05$ \\
\hline IVF-ET attempt & $2.00 \pm 1.41$ & $2.12 \pm 0.35$ \\
\hline Surgery due to acute abdomen & $6 / 29$ & $2 / 11$ \\
\hline Colchicine usage period (years) & $11.10 \pm 3.51$ & There is a story of using colchicine 2 years ago \\
\hline Total rFSH döșe & $2615.52 \pm 848.89$ & $2575.10 \pm 661.30$ \\
\hline rFSH usage time (day) & $9.21 \pm 1.35$ & $8.90 \pm 2.01$ \\
\hline Endometrial thickness (mm) & $10.70 \pm 1.56$ & $9.86 \pm 0.32$ \\
\hline FSH (IU/L) & $7.29 \pm 3.65$ & $6.87 \pm 1.47$ \\
\hline LH (IU/L) & $5.15 \pm 1.04$ & $4.93 \pm 0.56$ \\
\hline $\mathrm{E} 2(\mathrm{pg} / \mathrm{mL})$ & $49.55 \pm 15.41$ & $38.12 \pm 02.30$ \\
\hline P4 & $0.59 \pm 5.83$ & $0.48 \pm 3.12$ \\
\hline PRL & $30.45 \pm 9.91$ & $31.23 \pm 4.41$ \\
\hline E2 on hCG day & $2121.34 \pm 2069.21$ & $2035.12 \pm 1350.93$ \\
\hline P4 on hCG day & $0.71 \pm 0.50$ & $0.83 \pm 1.62$ \\
\hline Total oocyte & $10.76 \pm 9.97$ & $11.04 \pm 6.13$ \\
\hline MII oocyte & $7.24 \pm 5.72$ & $8.13 \pm 4.61$ \\
\hline Appearence of zona pellucida and COCs & Normal & Normal \\
\hline $2 \mathrm{PN}$ & $4.90 \pm 4.10$ & $5.01 \pm 3.23$ \\
\hline Clinical pregnancy rates & $15 / 29(51.7 \%)$ & $6 / 11(54.5 \%)$ \\
\hline Miscarriage & $3 / 15(20 \%)$ & $1 / 6(16.6 \%)$ \\
\hline Ongoing pregnancy rates & $12 / 29(41.3 \%)$ & $5 / 11(45.4 \%)$ \\
\hline Preterm birth rates & $3 / 29(10.3 \%)$ & $2 / 11(18.1 \%)$ \\
\hline Term birth rates & $9 / 29(31.0 \%)$ & $3 / 11(27.2 \%)$ \\
\hline Birth weight (g) & $2921.67 \pm 681.93$ & $2865.14 \pm 476.01$ \\
\hline
\end{tabular}


Table 2. Subfertility causes of both groups of FMF participants.

\begin{tabular}{|lcc|}
\hline & FMF patientsreceiving colchicine $(\mathbf{n}=\mathbf{2 9})$ & FMF patients withoutcolchicine $(\mathbf{n}=\mathbf{1 1})$ \\
\hline Appendectomy history & $6 / 29(20.6 \%)$ & $2 / 11(18.1 \%)$ \\
Laparoscopy history & $8 / 29(27.5 \%)$ & $3 / 11(27.2 \%)$ \\
Primary/secondary infertile & $22 / 7$ & $7 / 4$ \\
Miscarraige & $9 / 29(31.0 \%)$ & $2 / 11(18.1 \%)$ \\
Peritoneal ascite & $29 / 29(100 \%)$ & $11 / 11(100 \%)$ \\
Adhesion & $8 / 29(27.5 \%)$ & $3 / 11(27.2 \%)$ \\
Hydrosalpinx & $8 / 29(27.5 \%)$ & $1 / 11(9.09 \%)$ \\
Oligomenorrhea & $16 / 29(55.1 \%)$ & $3 / 11(27.2 \%)$ \\
PCOS & $3 / 29(10.3 \%)$ & $3 / 11(27.2 \%)$ \\
Diminished ovarian reserve & $10 / 29(34.4 \%)$ & $2 / 11(18.1 \%)$ \\
Unexplained infertility & $3 / 29(10.3 \%)$ & $3 / 11(27.2 \%)$ \\
Day 3 vsDay 5 ET & $19 / 10$ & $6 / 5$ \\
Fresh vs. thaw cycle & $17 / 12$ & $5 / 6$ \\
\hline
\end{tabular}

Ascites in the Douglas pouch was detected ultrasonographically or by laparoscopy in all patients in both groups. Pertioneal fluid was found in the ultrasonographic examination of the patients who did not undergo laparoscopy. Interestingly, we found that in patients who did not use colchicine, fluid accumulation overflows beyond the Douglas pouch and accumulates between the bowel loops. We did not do any analysis in the acid fluid, but the liquid image was clear and did not contain blood, possibly resembling the aseptic inflammatory reaction fluid developed due to FMF attacks.

After the hormonal evaluation and antral follicle count of both groups, we found three different types of pathologies that we thought caused subfertility. However, it is not possible to say clearly whether these pathologies are caused by the natural course of the disease or whether it is incidental. We can list these pathologies in order of frequency as follows; aligomenorrhea, decreased ovarian reserve and unexplained infertility. We detected PCOS in 3 of 16 cases diagnosed with oligomenorrhea. Decreased ovarian reserve was diagnosed in 10 cases receiving colchicine. In the group that did not take colchicine, ovulatory dysfunction was detected in 3 cases and decreased ovarian reserve in 2 case.

Clinical pregnancy rates were found to be significantly higher in patients who did not receive colchicine compared to patients who received colchicine (54.5\% vs $51.7 \%, \mathrm{p}<0.03)$. Although there was a slight tendency to increase in the patients who were given colchicine in terms of abortion rates, there was no significant difference between the two groups (20\% vs $16.6 \%$, p $<0.29$ ). There was a higher rate of ongoing pregnancy in the group that did not take colchicine compared to those who received colchicine, but it did not reach statistical significance ( $45.4 \%$ vs $41.3 \%$, p $>0.34$ ). Preterm birth rates were found to be significantly lower in the colchicine group compared to the control group (10.3\% vs $18.1 \%, \mathrm{p}<0.01)$. Term birth rates were also found to be significantly higher in colchicine group compared to patients not given colchicine ( $31.0 \%$ vs $27.2 \%$ ). There was no difference between the two groups in terms of birth weight.

\section{DISCUSSION}

Microtubule formation and subsequent spindle formation are pathways that have a critical role in both implantation and follicle development. Nucleating and organizing microtubules play an important role in cells that undergo both mitosis and meiosis. Microtubules are required for the preferential accumulation of transcripts and localization of mRNAs during oogenesis (10). Moreover, chromosome-directed spindle assembly has been observed in oogenesis and trofoblast transformation. Spindle formation begins with organization of microtubules around the human chromosomes (8). FMF patients are considered to be subfertile due to the nature of FMF disease and the mechanism of action of colchicine, the main drug used in its treatment. Impairment in both egg development and sperm count and function has been reported due to amyloid accumulation in the gonads or the blocking effect of colchicine on microtubule formation. However, the true cause of subfetility in FMF is unknown. While most authors blame the natural course of the disease, many authors blame colchicine $(3,4)$.

Colchicine shows its effect at the molecular level by its interaction with tubulin. This drug may have an important contribution to FMF-dependent subfertility as it inhibits meiosis and mitosis due to both its antimitotic activity and metaphase blocking effect (11). There were opinions in publications made before colchicine was put into use that there was amyloid accumulation in 
oocytes of FMF patients. It was thought that amyloid deposition thickened the zona and made fertilization difficult in spontaneous and IVF cycles $(2,3)$. However, the presence of amyloid in oocytes could not be clearly demonstrated in subsequent studies. Similarly, in our study, no difference was found in either the appearance or thickening of the cumulus oocyte complex (COC) or zona pellucida in FMF patients compared to patients without FMF. The lack of evidence of amyloid accumulation in oocytes may be due to the use of colchicine. However, both zona pellucida and COC of 11 FMF patients who did not receive colchicine treatment were normal in appearance and thickness. This result makes us think that colchicine use has no effect on amyloid accumulation in oocytes. Since ICSI is applied to all cases, even if there is amyloid accumulation, this does not pose a problem. In conclusion, as colchicine use has no effect on amyloide accumulation in oocytes, amyloid collection in zona pellucida or COC should be considered as a negligible culprit among the causes of subfertility due to FMF.

A main feature seen in all patients with or without colchicine is pertioneal fluid accumulation. Fluid accumulation in the Douglas cavity is a common condition in both ovulation and menstrual phases. However, the detection of peritoneal fluid accumulation in FMF patients regardless of the cycle period has raised questions about whether this fluid affects the fertility status of the patient. Peritoneal fluid accumulation was detected in both laparoscopy and ultrasonography in all of our patients using colchicine or not. However, in patients who did not take colchicine, the fluid accumulation spread between the bowel loops, while it was limited in the Douglas space in those who received colchicine. We did not analyze this fluid either bacteriologically or cytologically. However, previous studies have reported that the fluid shows aseptic inflammation conditions and does not contain bacteria and viruses. Less liquid in colchicine users may be due to the anti-inflammatory effect of the drug. However, we can state that pertioneal fluid accumulation does not have a significant effect on implantation and pregnancy rates, since there is no difference between IVF-ET results between patients who do not use colchicine and patients who use it. However, excessive fluid accumulation in spontaneous cycles may adversely affect fertility either by disrupting the ovulatory process or by reaching the endometrial cavity through the fallopian tubes. However, it is clear that our hypothesis needs to be clarified.

It has been reported that the use of colchicine causes an anti-inflammatory effect on the peritoneal and serosal surfaces, reducing adhesion formation (12) and contributing positively to fertility. Zayed et al (3) reported a moderate and severe adhesion rate of approximately $16 \%$ in FMF patients who underwent IVF-ET. Inconsistent with this data, in our study, adhesion frequency was found to be similar in both colchicine users $(27.5 \%)$ and those who did not $(27.2 \%)$. However, the fact that only two of the 8 cases who underwent adhesiolysis became pregnant suggests that adhesions due to FMF attacks do not have a serious effect on fertility outcome. All these data suggest that reduced fertility in FMF patients is a consequence of the natural course of the disease, independent of colchicine use and other detected causes such as adhesion and fluid accumulation.

Ovulatory dysfunction was detected in $27.2 \%$ of the patients who did not take colchicine, while this rate was $55.1 \%$ in those who received colchicine. Presence of high ovulatory dysfunction despite colchicine treatment suggests that there may be impairment in ovulatory functions within the natural course of FMF disease. We confirmed that the cause of ovulatory dysfunction was PCOS in three FMF patients. Similarly, the number of patients with decreased ovarian reserve was $10(34.4 \%)$ in the group receiving colchicine and $2(18.1 \%)$ in the group not receiving treatment. Interestingly, approximately one third of the studies in FMF patients reported both ovulatory dysfunction and a decrease in ovarian reserve $(13,14)$. Ehrenfeld et al $(15)$ classified the causes of subfertility in FMF patients as ovulatory dysfunction, peritoneal adhesions, and unexplained infertility. In the light of all these data, we can say that colchicine does not have a positive effect on ovarian reserve and ovulatory functions. As a result, the use of colchicine in FMF patients does not have a significant effect on both ovarian reserve and ovulatory functions. The continuation of ovulatory dysfunction in about half of the cases despite colchicine treatment supports this idea.

When the two groups were compared in terms of abortion rates, more miscarriages were encountered in colchicine patients than those who did not (20\% vs $16.6 \%)$. However, the difference was not significant. Our abortion rates in our patient groups are slightly lower than the literature data. Ehrenfeld et al (15) reported the abortion rate as $25 \%$ in FMF patients receiving colchicine treatment. In the light of these results, it would not be speculation to comment that taking colchicine treatment does not have a positive effect on early abortions. However, this opinion needs to be confirmed with studies containing a large number of cases.

The fact that the clinical pregnancy rate showed a significant increase in the group in which colchicine was not given compared to those given colchicine suggests that colchicine did not have a positive contribution on early embryo implantation. Similarly, the high rate of abortion in colchicine patients suggests that colchicine 
does not have a positive effect on endometrial receptivity during early pregnancy. Colchicine may contribute negatively to implantation by blocking cytotrophoblastsyncytiotrophoblast transformation through microtubule formation. However, this is an issue that requires further investigation. The positive effects of colchicine begin to emerge in the following weeks of pregnancy. The low preterm birth rates in colchicine patients suggested that this drug contributes to the continuation of pregnancy by positively modulating the endometrial inflammatory process. Increased local and systemic inflammatory processes due to FMF may be better regulated by the use of colchicine in the later stages of pregnancy. The higher term birth rates in patients using colchicine supports this view.

\section{CONCLUSION}

We investigated, for the first time, possible effects of colchicine use on IVF-ET results. FMF and colchicine treatment is relevant in IVF treatment in two aspects. One is the chronic recurring attacks causing infertility and two is continued use of colchicine during IVF process which can theoretically decrease IVF success. In this study, except for the positive effect on the number of term pregnancies, we could not detect any negative effect of the colchicine on the IVF-ET outcome. Colchicine did not have a clear effect on early embryo implantation and miscarriage rates. Increasing the number of term pregnancies by decreasing preterm birth rates suggests that colchicine plays an important role in the regulation of placentation in infertile FMF patients.

\section{ETHICAL DECLARATIONS}

Ethics Committee Approval: The study was carried out with the permission of the Research Ethics Committee of Erciyes University (Date: 09/09/2020, Decision No: 2020426).

Informed Consent: Verbal and written informed consent was obtained from all participants who participated in this study.

Referee Evaluation Process: Externally peer-reviewed.

Conflict of Interest Statement: The authors have no conflicts of interest to declare.

Financial Disclosure: The authors declared that this study has received no financial support.

Author Contributions: All authors declare that they have all participated in the design, execution, and analysis of the paper and that they have approved the final version.

\section{REFERENCES}

1. Migita K, Asano T, Sato S, Koga T, Fujita Y, Kawakami A. Familial Mediterranean fever: overview of pathogenesis, clinical features and management. Immunol Med 2018; 41: 55-61.

2. Cerquaglia C, Verrecchia E, Fonnesu C, et al. Female reproductive dysfunction in familial Mediterranean fever patients with and without colchicine treatment. Clin Exp Rheumatol 2010; 28: 101.

3. Zayed A, Nabil H, State O, Badawy A. Subfertility in women with familial Mediterranean fever. J Obstet Gynaecol Res 2012; 38: 1240-4.

4. Merlin HE. Azoospermia caused by colchicine. a case report. Fertil Steril 1972; 23: 180-1.

5. Ben-Chetrit E, Levy M. Colchicine: 1998 update. Semin Arth Rheum 1998; 28: 48-59.

6. Fitzgerald PH, Brehaut LA. Depression of DNA synthesis and mitotic index by colchicine in cultured human lymphocytes. Exp Cell Res 1970; 59: 27-31.

7. Haimov-Kochman R, Ben-Chetrit E. The effect of colchicine treatment on sperm production and function: a review. Hum Reprod 1998; 13: 360-2.

8. Holubcová Z, Blayney M, Elder K, Schuh M. Human oocytes. error-prone chromosome-mediated spindle assembly favors chromosome segregation defects in human oocytes. Science 2015; 348: 1143-7.

9. Goldfinger SE. Colchicine for familial Mediterranean fever. N Engl J Med 1972; 287: 1302.

10. Pokrywka NJ, Stephenson EC. Microtubules are a general component of mRNA localization systems in Drosophila oocytes. Dev Biol 1995; 167:363-370.

11. Handel M A. Effects of colchicine on spermatogenesis in the mouse. J Embriol Exp Morphol 1979; 51: 73-5.

12. Garcia-Gonzalez A, Weisman MH. The arthritis of familial Mediterranean fever. Semin Arthritis Rheum 1992; 22: 139-50.

13. Mamou H. Treatment of periodic disease with immunodepressive agents. Sem Hop 1970; 46: 2030-4.

14. Ismajovich B, Zemer D, Revach M, Serr DM, Sohar E. The causes of sterility in females with familial Mediterranean fever. Fertil Steril 1973; 24: 844-7.

15. Ehrenfeld M, Brzezinski A, Levy M, Eliakim M. Fertility and obstetric history in patients with familial Mediterranean fever on long-term colchicine therapy. Br J Obstet Gynaecol 1987; 94: 1186-91. 